\title{
A study on impact of patient counseling and education on quality of life in patients with metabolic syndrome
}

\author{
N.V. Prasanth*, V. Najiya Saheer, M. Naina Paul, P.K. Najla, K.P. Nizar Mohamed \\ Department of Pharmacy Practice, Al Shifa College of Pharmacy, Perinthalmanna, Kerala, 679325, India
}

\section{A R T I C L E I N F O}

\section{Keywords:}

Health related quality of life

Metabolic syndrome

Quality of life

\begin{abstract}
A B S T R A C T
Background: The components of Metabolic syndrome (MetS), have obvious consequences on Health Related Quality Of Life (HRQoL), the question whether metabolic syndrome is associated with the change of well-being and HRQoL requires evaluation.

Objective: The major objective of the study was to provide patient counseling and education on various aspects of metabolic syndrome to improve QoL of the patients.

Method: The study population was randomly categorized into control and intervention group. The intervention group was provided with patient counseling and Patient Information Leaflets along with usual care. The control group received the usual care provided by the doctors, nurses and technicians. The QoL of both groups was assessed using modified WHO-BREF QOL questionnaire during the subsequent follow-up.

Results: It was found that there is a statistical significant difference in the physical, psychological and social domain values in the test group at baseline and after intervention phase with t value $-10.574,-10.796$ and $-2.221(\mathrm{p}=0.001, \mathrm{p}=0.001 \& \mathrm{p}=0.031)$.

Conclusion: In the present study, there is an improvement in the QoL scores of the intervention group compared to that of the control group. Clinical pharmacist's service can be very beneficial for the management and monitoring of MetS patients to improve their QoL.
\end{abstract}

\section{Introduction}

Metabolic syndrome (MetS), characterized by central obesity, dyslipidemia, hyperglycemia and hypertension is currently a major global public health challenge because it involves a serious risk of cardiovascular disease and type 2 diabetes. ${ }^{1}$ Definition of metabolic syndrome by National Cholesterol Education Program's Adult Treatment Panel III (NCEP: ATP III) Criteria, 2001 includeany three of the following:1. Central obesity: Waist circumference $>102 \mathrm{~cm}$ or 40 inches in males, $>88 \mathrm{~cm}$ or 35 inches in females, 2 . Hypertriglyceridemia: Triglycerides $\geq 1.7 \mathrm{mmol} / \mathrm{L}(150 \mathrm{mg} / \mathrm{dl})$, 3 . Low HDL cholesterol: $<1.0$ $\mathrm{mmol} / \mathrm{L}(40 \mathrm{mg} / \mathrm{dl})$ in males, $<1.3 \mathrm{mmol} / \mathrm{L}(50 \mathrm{mg} / \mathrm{dl})$ in females, 4.Hypertension: Blood pressure $\geq 135 / 85 \mathrm{mmHg}$ (or treated for hypertension), 5. Fasting plasma glucose $\geq 6.1 \mathrm{mmol} / \mathrm{L}(110 \mathrm{mg} / \mathrm{dl}){ }^{2}$ According to International Diabetes Federation (IDF) criteria, 2006 metabolic syndrome is defined as Central obesity (defined as waist circumference $\geq 94 \mathrm{~cm}$ for males or $\geq 80 \mathrm{~cm}$ for femalesPlus any two of the following: 1.Raised triglycerides $>1.7 \mathrm{mmol} / \mathrm{L}(150 \mathrm{mg} / \mathrm{dl})$ or specific treatment for this lipid abnormality, 2. Reduced HDL cholesterol: $<1.03 \mathrm{mmol} / \mathrm{L}(40 \mathrm{mg} / \mathrm{dl})$ in males or $1.29 \mathrm{mmol} / \mathrm{L}(50 \mathrm{mg} / \mathrm{dl})$ in females or specific treated for this lipid abnormality, 3.Raised blood pressure $\geq 130 / 85 \mathrm{mmHg}$ or treated for previously diagnosed hypertension, 4. Raised fasting plasma glucose(FPG): $>5.6 \mathrm{mmol} / \mathrm{L}$ $(100 \mathrm{mg} / \mathrm{dl})$ or previously diagnosed type 2 diabetes. If FPG is greater than $5.6 \mathrm{mmol} / \mathrm{L}$ or $100 \mathrm{mg} / \mathrm{dl}$, an oral glucose tolerance test is strongly recommended, but it is not necessary to define metabolic syndrome. If BMI is greater than $30 \mathrm{~kg} / \mathrm{m}^{2}$, central obesity can be assumed and waist circumference does not need to be measured. ${ }^{3}$ (see Tables 1-3, Figs. 1-7).

The prevalence of metabolic syndrome has increased in developing countries in recent decades. It is estimated that around $20-25 \%$ of the world's adult population have the metabolic syndrome and they are twice as likely to die from and three times as likely to have a heart attack or stroke compared with people without the syndrome. In addition, people with metabolic syndrome have a 5 fold greater risk of developing type 2 diabetes. ${ }^{4}$ Approximately about one third of urban south Asians have evidence of the metabolic syndrome. In urban Indian adults, the prevalence $(41.1 \%)$ is high compare to rural area. Moreover, insulin resistance was observed to the therein nearly $30 \%$ of Asian Indian children and adolescent and many exhibit features of metabolic

\footnotetext{
* Corresponding author. Dept of Pharmacology, Al Shifa college of Pharmacy, Poonthavanam P.0, Perinthalmanna, Kerala, 67932, India.

E-mail address: nv.prasanth@gmail.com (N.V. Prasanth).
} 
Table 1

Prevalence of metabolic syndrome.

\begin{tabular}{lll}
\hline Category & Frequency & Percentage \\
\hline Met-S & 658 & $29.44 \%$ \\
Non Met-S & 1577 & $70.55 \%$ \\
Total & 2235 & $100 \%$ \\
\hline
\end{tabular}

It is clear from the table that the percentage of patients having MetS (29.44\%) compared to patients not having MetS. In this study the prevalence of MetS was found to be $29.44 \%$.

Table 2

Comparison of pre-interventional qol between two groups.

\begin{tabular}{lllll}
\hline \multirow{2}{*}{ Domains } & Intervention group & Control group & \multirow{2}{*}{ t value } & \multirow{2}{*}{ P value } \\
\cline { 2 - 3 } & Mean $\pm \mathrm{SD}$ & Mean $\pm \mathrm{SD}$ & & \\
\hline Physical health & $27.78 \pm 4.70$ & $21.90 \pm 3.13$ & 7.357 & 0.001 \\
Psychological & $23.68 \pm 4.19$ & $16.94 \pm 2.19$ & 10.060 & 0.001 \\
Social relationships & $9.56 \pm 2.13$ & $9.88 \pm 1.62$ & -.842 & 0.402 \\
Environmental & $24.88 \pm 4.19$ & $24.66 \pm 3.54$ & 0.283 & 0.778 \\
\hline
\end{tabular}

Table 3

Comparison of post interventional qol between two groups.

\begin{tabular}{lllll}
\hline \multirow{2}{*}{ Domains } & Intervention group & Control group & \multirow{2}{*}{ t value } & \multirow{2}{*}{ P value } \\
\cline { 2 - 4 } & Mean $\pm \mathrm{SD}$ & Mean $\pm \mathrm{SD}$ & & \\
\hline Physical health & $20.90 \pm 2.88$ & $21.60 \pm 3.09$ & -1.169 & 0.245 \\
Psychological & $17.48 \pm 2.41$ & $17.00 \pm 2.15$ & 1.047 & 0.297 \\
Social relationships & $9.54 \pm 2.11$ & $9.92 \pm 1.72$ & -.986 & 0.327 \\
Environmental & $24.72 \pm 4.32$ & $24.74 \pm 3.64$ & -.025 & 0.980 \\
\hline
\end{tabular}

syndrome. ${ }^{5}$ Kerala, the diabetic capital of India has a high obesogenic environment. Several studies showed a prevalence of $89.7 \%$ obesity among patients with metabolic syndrome. A hospital based study done by Jyothi B et al. showed a prevalence of metabolic syndrome (66.2\%). The high prevalence, poor detection and control of metabolic disorders in Kerala necessitate need for better therapeutic measures and patient education. ${ }^{6}$

There are many interrelated factors that are thought to be important in the development of metabolic syndrome. ${ }^{7}$ The major risk factors include age $>50$ years, female sex, positive family history, smoking, non-vegetarian diet, sleep inadequacy, stress and alcohol intake. ${ }^{8,9}$

The primary goal of clinical management in individuals with the metabolic syndrome is to reduce risk for clinical atherosclerotic disease. Even in people with the metabolic syndrome, first-line therapy is directed toward the major risk factors: LDL-C above goal, hypertension, and diabetes. ${ }^{10}$

\section{Methodology}

A prospective interventional randomized control trial was carried out for a period of six months in the General Medicine II department of a tertiary care referral hospital at Malappuram, Kerala.

National Cholesterol Education Program's Adult Treatment Panel III (NCEP: ATP III) Criteria, 2001 and International Diabetes Federation (IDF) criteria, 2006 were used to define metabolic syndrome.

The study was approved by the institutional ethics committee of KIMS-AL SHIFA Hospital. It was ensured that work described has been carried out in accordance with The Code of Ethics of the World Medical Association (Declaration of Helsinki).

\subsection{Informed consent}

The nature, type and intention of the study were explained to the subjects by direct interaction. Participants were given sufficient time to decide whether or not to participate in the study. Informed consent was obtained for experimentation with human subjects. The privacy rights of human subjects must always be observed.

\subsection{Sample size}

A total of 136 patients were used as subjects for the study that included 66 subjects in the control group and 70 subjects in the case group.

\subsection{Inclusion criteria}

- All patients visited the general medicine II department with condition of metabolic syndrome.

- Patients above age $\geq 20$ years.

- Patients not having any known cardiovascular diseases.

\subsection{Exclusion criteria}

- Pregnant females.

- Patients of age $<20$ years.

- Patients having existing cardiovascular diseases.

For the study. WHOQOL-BREF questionnaire was used to assess quality of life of patients, as it is the most widely accepted scale in this regard. The questionnaire was translated to the local language Malayalam and validated.

It includes 26 questions divided among 4 domains.

The 4 domains include physical health, Psychological, Social relationship and Environmental. Framingham risk score was used to assess the 10 year cardiovascular risk of patients with metabolic syndrome. The Framingham risk score for 10 year CVD risk assessment ranged from score of $<1$ to $>30$. If FRS is $\geq 20 \%$ then the subjects will have higher risk of developing CVD in the next 10 years. If FRS is 10-19\% people will have intermediate risk for developing CVD and if its $<10 \%$ risk will be low.WHO/ISH risk prediction chart is used to evaluate the 10 year cardiovascular risk of patients with metabolic syndrome. These charts indicate 10-year risk of a fatal or non-fatal major cardiovascular event (myocardial infarction or stroke), according to age, sex, blood pressure, smoking status, total blood cholesterol and presence or absence of diabetes mellitus. A separate patient information leaflet was prepared regarding the diet and lifestyle modification to counsel and educate the patients.

In the pre-interventional phase, demographic data and complete history of patients was obtained from case reports, medication charts, and personal interviews. Quality of life of subjects was evaluated using WHO-BREF QOL questionnaire. 10 year CVD risk of patients was evaluated by using Framingham risk score and WHO/ISH risk prediction charts. The study population was randomized by statistician using randomized block design.

In the interventional phase, counseling to the intervention group was performed outside the OP in a separate area to ensure privacy. For the inpatients, bedside counseling was given. Each counseling session was about 7-10 min depending on the patients. The counseling program includes the information about the disease, treatment goals, and importance of medication adherence, possible adverse drug reactions, management of the disease and life style modifications. Patient education was done by using patient information leaflet. And the control group received the usual care provided by the doctors, nurses and technicians only. The patient counseling, education and PILs were provided to this group at the end of the study.

Phase 3 mainly consists of follow up visits. Most of the patients were followed up for next subsequent appointments, to re-assess their quality of life. Some patients were followed up by telephonic interviews. Data collected from the study was tabulated in Microsoft Excel 2010 and 


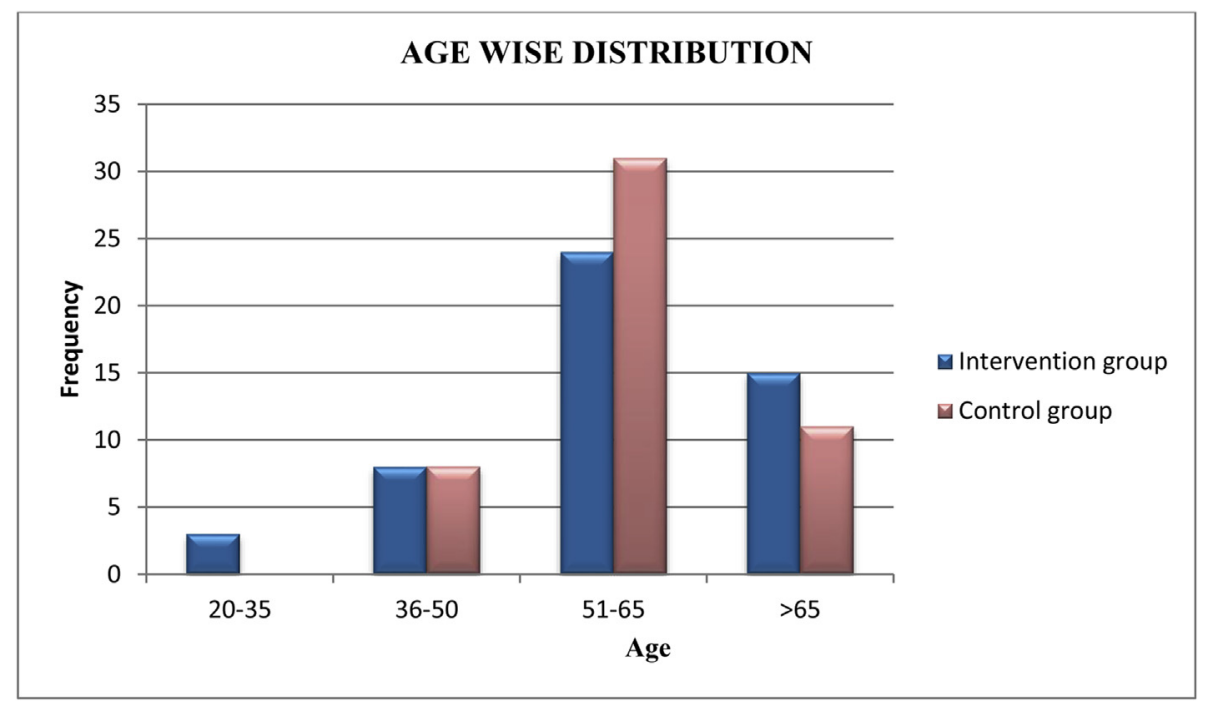

Fig. 1. Age wise distribution.

Majority of the subjects enrolled in the study were in the age group of 51-65 $(\mathrm{N}=55)$ followed by $>65(\mathrm{~N}=26)$.

On using Chi-square test there was no significant difference in the age among intervention group and control group with $\mathrm{p}=0.212\left(\chi^{2}=4.506\right)$.

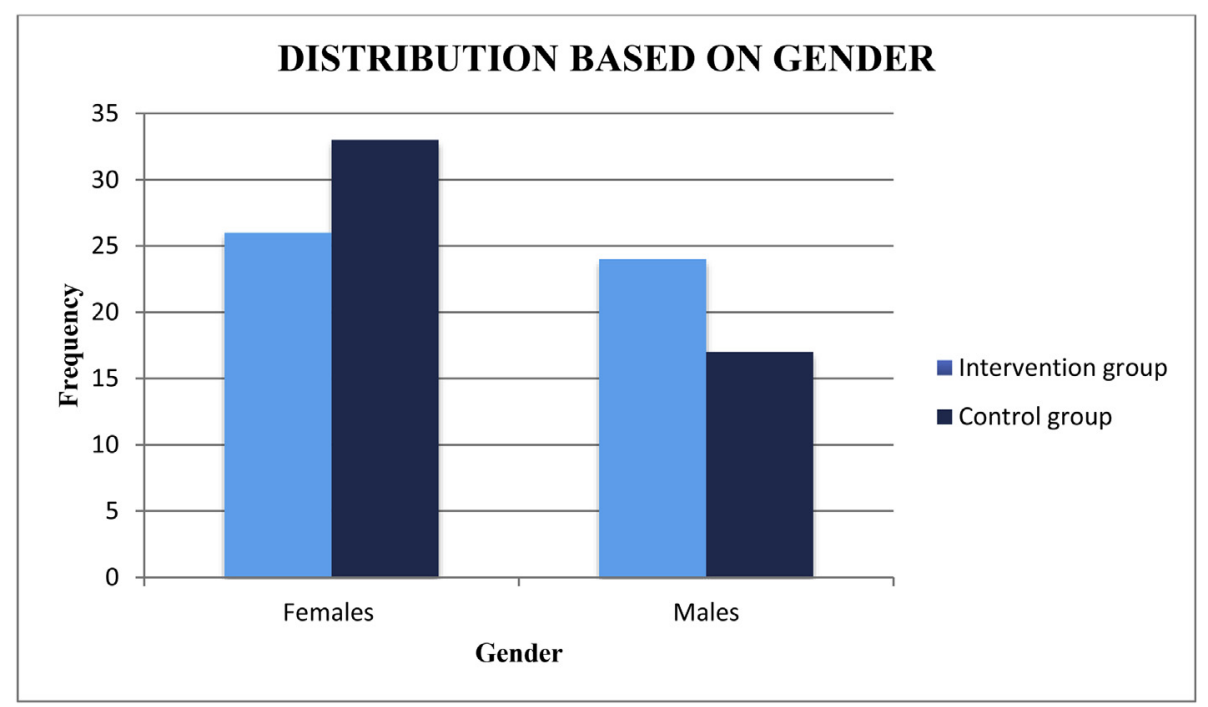

Fig. 2. Sex wise distribution.

On using Chi-square test there was no significant difference in gender distribution between control group and intervention group with $\mathrm{p}=0.155\left(\chi^{2}=2.026\right)$.

were keyed into the Statistical Package for Social Science (SPSS Inc. Chicago, II, USA) computer software version 20 for windows and analyzed by appropriate statistical methods. Statistical analysis was both descriptive at $95 \%$ confidence level. $\chi^{2}$ test was used for testing association of qualitative variables in each group of the study. Independent $t$-test was used to compare quantitative variables between control and intervention group of the study. Demographic variables were analyzed using paired $t$-test. A two-tailored probability value of $<0.05$ was considered to be statistically significant.

Deliberate attempts were made to prevent the dropout of subjects from the study. This included frequent direct and telephonic interaction with the patients, educating the subjects about the importance of the study, and maintenance of a friendly environment etc.

\section{Results}

A total of 136 Metabolic Syndrome patients without cardiovascular co-morbidities were enrolled in the study (out of the 658 patients with metabolic syndrome according to NCEP-ATP III and IDF criteria). So the prevalence of metabolic syndrome was $29.44 \%$ in this study In the preintervention phase, 70 patients received intervention and 68 patients were taken as control group. Only 50 patients from intervention group and 50 patients from control group showed up for review. So that 36 missing subjects were excluded from post-intervention phase.The baseline characteristics were similar in both groups.

It was found that most of the study subjects are coming under the age group of 51-6 5(55\%). The prevalence of MetS was higher in female patients and lower in male patients (41\%). Most of the patients were found to be non-vegetarian (85\%). It is clear from the table that most of the patients have a positive family history (76\%). In adequate physical activity (69\%) was found to be associated with the prevalence of MetS. Mean CVD risk by WHO/ISH method was found to be 3.5 in intervention group and 3.26 in the control group. The mean difference was found to be 0.24 . On using independent $t$-test, there was no significant difference in the risk level among intervention group and control group with $\mathrm{p}=0.159$ ( $\mathrm{t}$ value $=0.159$ ). Mean CVD risk by FRS method was found to be 2.7 in the intervention group and 2.74 in the control group. The mean difference was found to be -.04 . On using independent $t$ - 


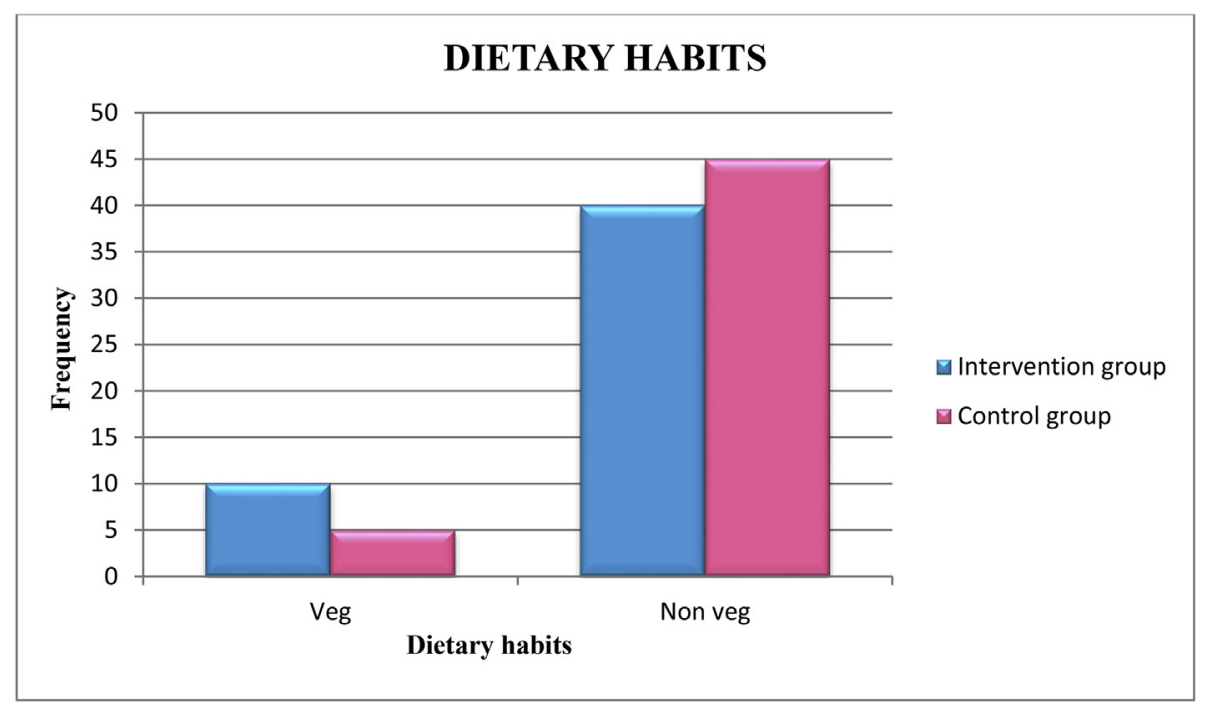

Fig. 3. Distribution based on dietary habits.

Dietary habits in both case group and intervention group with $\mathrm{p}=0.161\left(\chi^{2}=1.961\right)$.

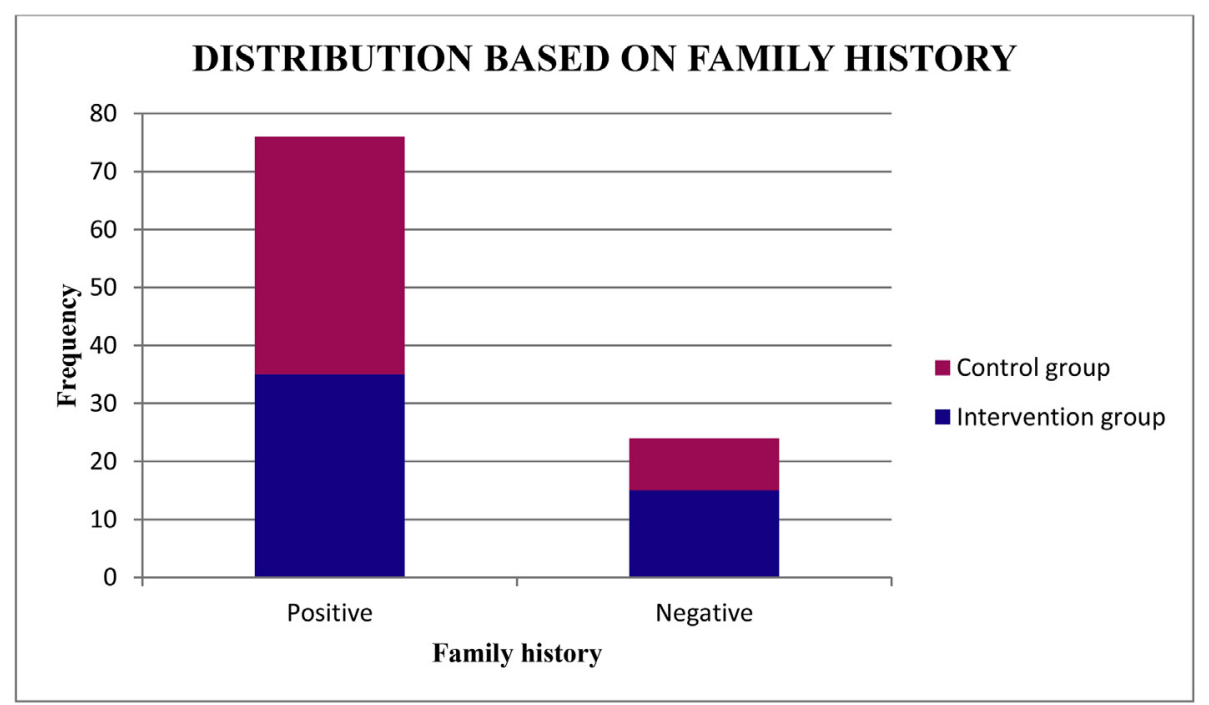

Fig. 4. Distribution based on family history.

The difference in family history in intervention group and control group was found to be non-significant with $\mathrm{p}=0.160\left(\chi^{2}=1.974\right)$.

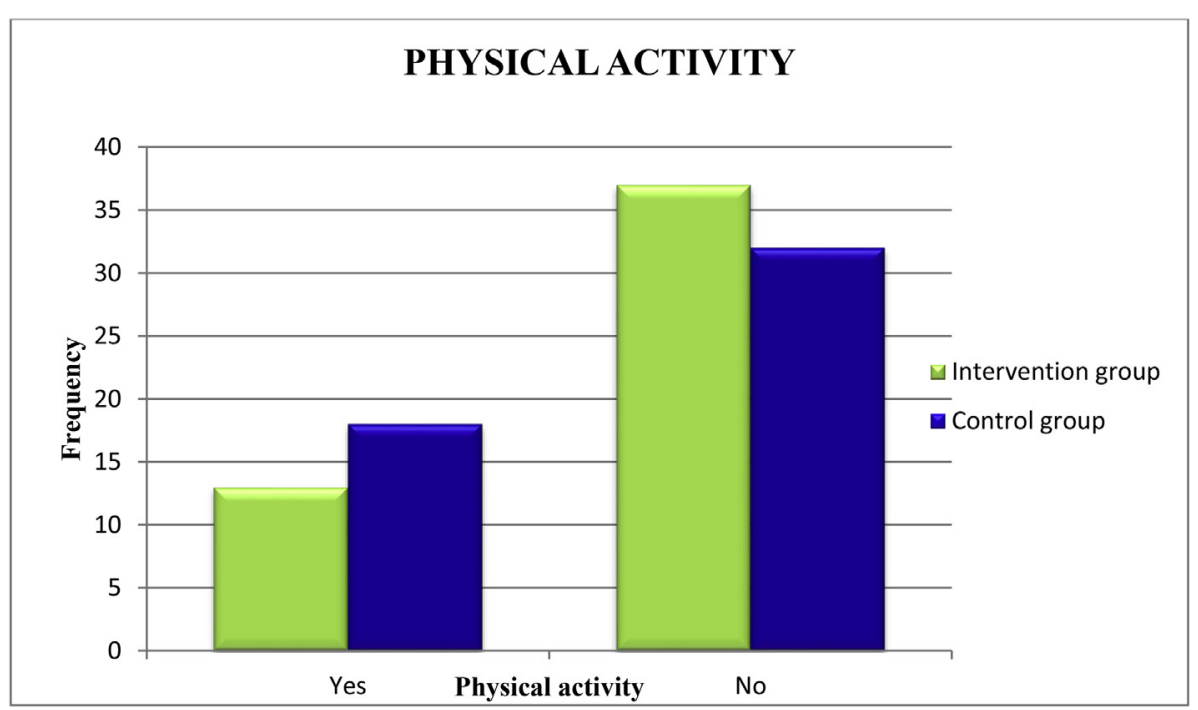

Fig. 5. Distribution based on physical activity.

There was no significant difference in the physical activity among control group and intervention group with $\mathrm{p}=0.280\left(\chi^{2}=1.169\right)$.

The prevalence of MetS was higher in female patients and lower in male patients (41\%). Most of the patients were turned to be non-vegetarian (85\%). It is clear from the table that most of the patients have a positive family history (76\%). In adequate physical activity (69\%) was found to be associated with the prevalence of MetS. 


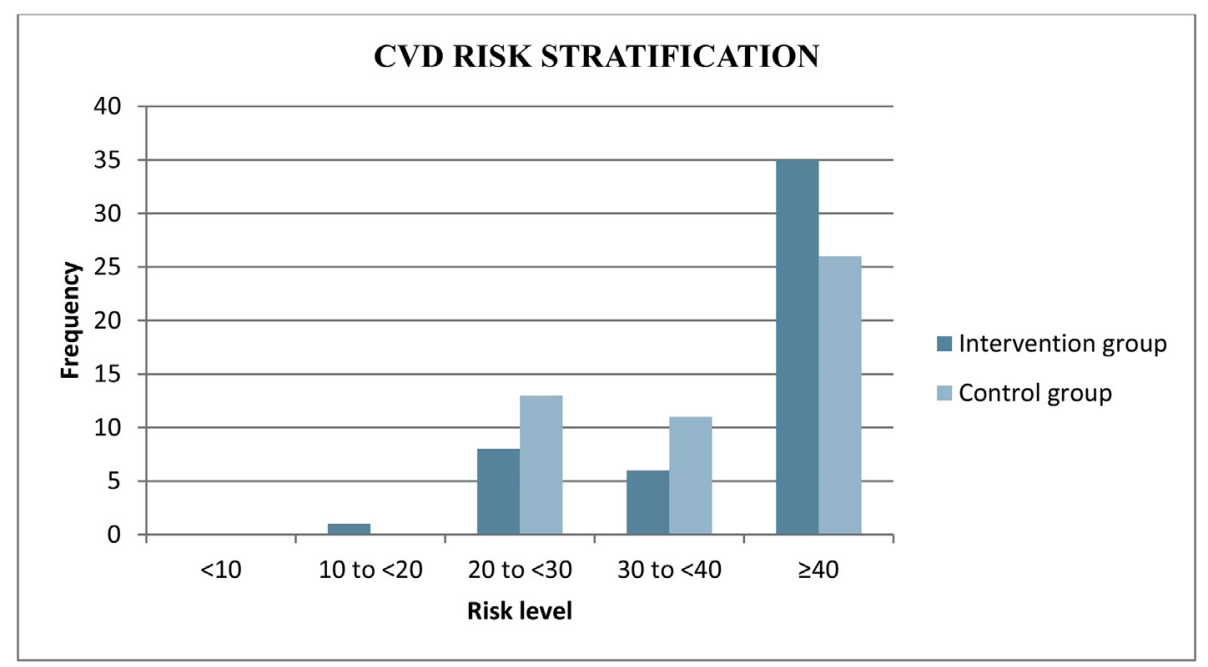

Fig. 6. CVD risk stratification; WHO/ISH method.

Among the total study population, $61 \%(n=61)$ of the subjects were found to be having a higher risk of developing cardiovascular diseases.

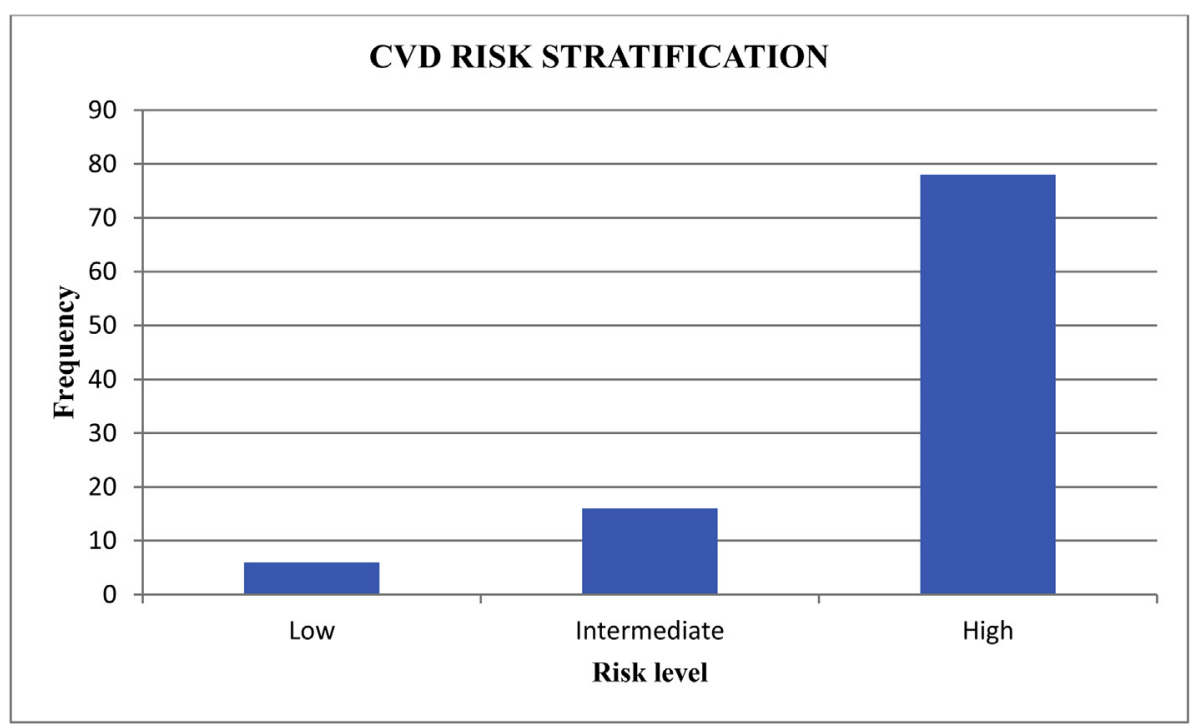

Fig. 7. CVD risk stratification; FRS method. Mean CVD risk by WHO/ISH method was found to be 3.5 in intervention group and 3.26 in the control group. The mean difference was found to be 0.24 . On using in dependent $t$-test, there was no significant difference in the risk level among intervention group and control group with $\mathrm{p}=0.159$ ( $\mathrm{t}$ value $=0.159$ ).

test, it was found that there is no significant difference in the CVD risk level among study populations with $\mathrm{p}=0.728(\mathrm{t}=-.349)$.

On using paired $t$-test, it was found that there is a statistical significant difference in the domain $1,2 \& 4$ values at baseline and during follow up with $t$ value $-10.574,-10.796$ and $-2.221(\mathrm{p}=0.001$, $\mathrm{p}=0.001 \& \mathrm{p}=0.031$ ). And there was no significant difference in the domain 3 with $\mathrm{t}$ value $-1.00(\mathrm{p}=0.322)$. On using paired $t$-test, it was found that there is no significant difference in the 4 domains among control group at baseline and during follow up with t values -1.439 , $0.518,-.903,0.076$ respectively $(\mathrm{p}=0.157, \mathrm{p}=0.607, \mathrm{p}=0.369$, $\mathrm{p}=0.940$ ).

On using independent $t$-test, it was found that the two groups are homogenous in terms of quality of life. There was no statistical significant difference in the physical, psychological, social and environmental domains between intervention and control groups with $t$ values $-1.169,1.047,-.986,-.025$ respectively ( $p>0.05$ ).In the post interventional phase, there was a statistically significant difference in the physical and psychological domains between intervention and control groups with $\mathrm{t}$ values 7.357 and 10.060 ( $\mathrm{p}<0.05$ ). And there was no significant difference in the other two domains with $\mathrm{t}$ values -.842 and $0.283(\mathrm{p}=0.402$ and $\mathrm{p}=0.778)$.

\section{Discussion}

Our study found out that the prevalence of MetS was found to be $29.44 \%$ which can be correlated to the prevalence rate $(41.1 \%)$ of the study conducted by D.S Prasad et al. ${ }^{5}$

Majority of the subjects enrolled in the study were in the age group of $51-65(\mathrm{~N}=55)$ followed by $>65(\mathrm{~N}=26)$. A significant increase in the frequency of MetS after the age of 50 years was observed in concordance with results of a study conducted by Jaspinder kaur et al. ${ }^{13}$ An overall increasing trend with advancing age might be contributed an evolution of insulin resistance, hormonal alterations, and increase in visceral adipose tissue with age.

A higher trend of MetS in females (59\%) than in males(41\%) in the present study was found to be supported by Maria aguilar et al. ${ }^{11}$ shows women(35.6\%) and men(30.3\%).Women transition from the pre-menopause to post-menopause state with substantial metabolic changes and estrogen deficiency might lead them to an increased predisposition to MetS.

Concerning drinking, drinking more than once per week increased the prevalence of MetS according to a similar study conducted by Eun Shil Lim et al. ${ }^{12}$ But, in our study, $84 \%$ of the subjects were non-alcoholic. This can be explained by a higher rate of women in the study 
population.

With regard to smoking status, smokers having an increased prevalence of MetS, in accordance with a study conducted by Hyung-Sik Kong et al. ${ }^{13}$ which shows $51.8 \%$ of workers in medium-scale enterprises were current smokers, $49.8 \%$ in small-scale and $43.9 \%$ in large-scale enterprises. In this study, $77 \%$ of subjects were ex/nonsmokers.

Sleep inadequacy (adequate sleep time was taken as 7-8 h) had further contributed to the diagnosis of MetS in our study similar to the study conducted by Jaspinder Kaur et al. ${ }^{8}$ Our study found out that $48 \%$ of the subjects were suffering from inadequate sleep.

The effect of exercise was also significant, inadequate physical activity $(69 \%)$ was found to be associated with the prevalence of MetS, which can be correlated with a study conducted by Eun Shil Lim et al. ${ }^{12}$ which shows prevalence of Mets was $41.6 \%$ among non-exercisers than in exercisers $39.4 \%$.

A positive family history has shown predisposition to MetS than a negative family history in current study. In our study, most of the patients have a positive family history (76\%) which was supported by Jainder Kaur et al. ${ }^{8}$ in another similar study.

The dietary patterns had marked differences in the prevalence of MetS. Most of the patients were turned to be non-vegetarian (85\%) almost similar to study conducted by Nico S Rizzo et al. ${ }^{14}$ that shows the MetS was highest in non-vegetarians (39.7\%), intermediate in semi vegetarians (37.6\%) and lowest in vegetarians (25.2\%).

The Framingham risk score for 10 year CVD risk assessment ranged from score of $<1$ to $>30$. If FRS is $\geq 20 \%$ then the subjects will have higher risk of developing CVD in the next 10 years. Based on the Framingham risk score, the subjects were divided into low, intermediate and high risk, overall the prevalence 10 year risk of CVD for patients with MetS was significantly different with those without MetS comparable to a study conducted by Gholamreza yousefzadeh et al. ${ }^{15}$ which shows (74.3 vs. $86.4 \%$ ) for low risk patients, (18.1 v/s $12.3 \%$ ) for intermediate risk patients and (7.6 v/s 1.3\%) for high risk patients.

The QoL among MetS patients was reduced as correlated to healthy population as stated by the study conducted by Mahesh V et al. ${ }^{16}$.

The impact of patient counseling and Patient education in the QoL scores among diabetes by Adepu $\mathrm{R}$ et al. ${ }^{17}$ and hypertensive patients by Biradar SS et al. ${ }^{18}$ significantly improved in the intervention group compared to the control group over a period of 6 months. In the present study there is an improvement in the QoL scores of the intervention group compared to that of the control group.

\section{Conclusion}

Metabolic syndrome is a major and escalating public health and clinical challenge worldwide due to urbanization, increasing obesity and sedentary life habits. Metabolic syndrome confers a twofold risk of developing CHDs over the next 5-10 years. The components of metabolic syndrome, such as abdominal obesity, hypertension and hyperglycemia impair quality of life which leads to functional dependence. Although, obesity and diabetes have obvious consequences on HRQoL, the question whether metabolic syndrome is associated with the change of well-being and HRQoL requires further evaluation.

The outcome of the study highlights the need of care and patient education programme for the improvement of the QoL of MetS patients. This care should be extended after discharge of patients from the hospital and maintained throughout their follow-up period. Provision of multi-disciplinary care will enable the improvement of QoL in MetS patients. In addition, lifestyle changes should be made by the patients themselves to have better clinical output. Clinical pharmacist's service can be very beneficial for the management and monitoring of MetS patients to improve their QoL.

\section{Limitations of the study}

The limitations for the present study is that the duration of study is very less and is not sufficient for the assessment of impact of Patient Education and Patient Counseling in QoL of MetS patients. Only small sample size was taken for the study and this sample size cannot outline the QoL of the total MetS patients.

\section{Declarations of interest}

None.

\section{References}

1. Sharma Mahak, Mahra Ranjana. Obesity, metabolic syndrome and physical activity in indian adults. J Metab Syndrome. 2012;1:4

2. Executive summary of the third report of the National Cholesterol Education Program (NCEP) Expert Panel on Detection. Evaluation, and treatment of high blood cholesterol in adults (adult treatment Panel III). J Am Med Assoc. 2001;285:2486-2497.

3. The IDF consensus worldwide definition of the metabolic syndrome [http://www.idf org]. Accessed sept 21, 2006.

4. Borch Knut, Johnsen. Epidemiology of the metabolic syndrome. The Metabolic Syndrome. 2013;3:1331-1338.

5. Prasad DS, Kabir Z, Dash AK, Das BC. Prevalence and risk factors for metabolic syndrome in Asian Indians: a community study from urban Eastern India. $J$ Cardiovasc Dis Res. 2010;3:204-211.

6. Srinivasan Shubha, Lingegowda Jyothi B, Rajan Chinnappa, Prakash H, Muddegowda, Ramkumar Kurpad R. Metabolic syndrome in rural Kerala: a hospital based study. International Journal of Advances in Medicine. 2016;3(4):898-904.

7. Standl Eberhard. Aetiology and cosequences of the metabolic syndrome. Eur Heart $J$ Suppl. 2005;7:10-13.

8. Kaur J. Assessment and screening of the risk factors in metabolic syndrome. Med Sci. 2014;2(3):140-152.

9. Lim ES, Ko YK, Ban KO. Prevalence and risk factors of metabolic syndrome in the Korean population-Korean national health insurance corporation survey 2008. J Adv Nurs. 2013;69(7):1549-1561.

10. Grundy SM, et al. Diagnosis and management of the metabolic syndrome. Circulation. 2005;112(17):2735-2752.

11. Aguilar Maria, et al. Prevalence of the metabolic syndrome in the United States, 2003-2012. JAMA, J Am Med Assoc. 2015;313(19):1973-1974.

12. Lim Eun Shil, Ko Yu Kyung, Ban Keum Ok. Prevalence and risk factors of metabolic syndrome in the Korean population - Korean national health insurance corporation survey. J Adv Nurs. 2012;21:1549-1561.

13. Kong Hyung-sik, et al. Factors associated with metabolic syndrome and related medical costs by the scale of enterprise in korea. Ann Occup Environ Med. 2013;25(23):1-10.

14. Rizzo Nico S, et al. Vegetarian dietary pattern are associated with a lower risk of metabolic syndrome. Diabetes Care. 2011;34(5):1225-1227.

15. Yousefzadeh Gholamreza, et al. Applying the Framingham risk score for prediction of metabolic syndrome: the kerman coronary artery disease risk study, Iran. ARYA Atheroscler. 2015;11(3):179-185.

16. Mahesh V, et al. Quality of life in diabetic subjects with respect to metabolic syndrome: a case control study. International Journal of Community Medicine and Public Health. 2016;3(9):2393-2396.

17. Adepu R, Rasheed A, Nagavi BG. Effect of patient counseling on quality of life in typ2 diabetes mellitus patients in two selected South Indian community pharmacies. A study. 2007;69(4):519-524.

18. Biradar SS, Kapate R, Srinivas R, Raju SA. Assessment of pharmacist mediated patient counseling on hypertensive compliance with quality of life in south indian city. Int Res J Pharm. 2012;3(6):206-209. 